\title{
Application of prenatal ultrasonography and magnetic resonance imaging on fetal agenesis of corpus callosum
}

\author{
Ai-Ping Min'1 ${ }^{1}$, Li-Hua Zou² (1) \\ ${ }^{1}$ Department of Gynecology and Obstetrics, People's Hospital of Leshan Sichuan Province, Leshan, China \\ ${ }^{2}$ Department of Medical imageology, City Central Maternal and Child Health Hospital of Leshan, China
}

\begin{abstract}
Objectives: To evaluate the diagnostic value and clinical application of prenatal ultrasonography (US) and Magnetic Resonance Imaging (MRI) for different types of fetal Agenesis of the Corpus Callosum (ACC).

Material and methods: There were 42 cases of fetal ACC discovered by routine US, including complete ACC 18 cases and partial ACC 24 cases, checked by MRI within 1 week. The results were confirmed by head ultrasound after birth or brain biopsy after labor induction.

Results: From prenatal ultrasonic diagnosis, 18 cases were complete ACC and 24 cases were partial ACC. MRI was able to find complete ACC in 11 cases, partial ACC in 16 cases, and non-ACC in 15 cases. Labor induction or birth confirmed that, 11 cases were complete ACC, 14 cases were partial ACC, and 17 cases were non-ACC.The results of different types of ACC were detected by ultrasound and MRI were statistically significant $(p<0.05)$.MRI examination was superior to ultrasound in specificity, positive predictive value, negative predictive value, Youden index, and diagnostic index.

Conclusions: MRI is high specific degrees, diagnostic performance is satisfactory, should be use as a necessary method for prenatal definitive diagnosis of ACC. However, prenatal ultrasound can be tested repeatedly and can be combined with blood flow imaging detection in real time, and it is still the preferred method for screening fetal structural malformation in a comprehensive way, which is suitable for general screening of ACC.
\end{abstract}

Key words: agenesis of corpus callosum; ultrasonography; magnetic resonance imaging; prenatal diagnosis

Ginekologia Polska 2020; 91, 3: 132-136

\section{INTRODUCTION}

Agenesis of corpus callosum (ACC) is a rare congenital central nervous system malformation, with the incidence rate of about $0.3-0.7 \%$, and often associated with other fetal malformations [1,2]. ACC can be divided into complete and partial type in morphology, and also Isolated and Compound type with or without other malformations [3,4], different types of ACC have huge difference in prognosis. The precise function of the corpus callosum is not clear yet, which is currently believed to mainly connect the integrated bilateral motor language centers, motor ataxia area and audio/visual area and other areas [4], and often related to pathogenesis of autism, epilepsy, schizophrenia, dementia and other diseases [2]. High-resolution color Doppler ultrasound is the most important means of prenatal screening for ACC, while the rapid development of fetal magnetic resonance imaging (MRI) has provided a new approach for fetal intracranial studies, with high image resolution and free from the influence of factors such as the position of fetus. The two detection methods have significant differences. This study aims to explore the diagnostic value and application of prenatal ultrasound and magnetic resonance imaging for different types of ACC.

\section{Objectives}

The aim of the study was to compare the diagnostic value and clinical application of prenatal ultrasonography(US) with Magnetic Resonance Imaging (MRI) for different types of fetal Agenesis of the Corpus Callosum (ACC), and Provide reference for the formation of standardized consensus of prenatal diagnosis of ACC.

\section{MATERIAL AND METHODS}

A total of 42 cases of fetal ACC in ultrasonic or MRI diagnosis in our Hospitals from January 2013 to October 
2017 were performed with fetal magnetic resonance imaging examination within one week after the ultrasonic diagnosis, and followed up to labor induction or birth, maternal age $19 \sim 45(29.7 \pm 4.66)$ years, gestational age $21 \sim 39$ (28.96 \pm 4.43$)$ weeks. This study was approved and supervised by the medical ethics committee of the hospital.

Color Doppler ultrasound diagnostic instrument (GE Voluson 8, Accuvix A30), with convex array probe, and the frequency of $2.5 \sim 8.0 \mathrm{MHz}$ were used on ultrasound tests. Informed consent form was signed with the pregnant women before the examination. After examining the fetus according to the system ultrasonic inspection norms, scanning of the craniocerebral cross-section for each layer from the top to the base of the skull was performed for the intracranial abnormal fetuses. Scanning of the craniofacial sagittal section and coronal section was further performed if possible, with careful observation of intracranial arterial blood flow, assessment and classification of suspected cases of ACC, and storage of the related information at the same time.

MRI detected by superconducting magnetic resonance imaging instrument (GE HDX 1.5 T), with 8-channel abdominal surface coils. An informed consent form was signed before the examination. Sequence SS FSE T2W1 and FIESTA coronal, sagittal and axial three-directional scanning was adopted, the fetus was scanned according to fetal size and maternal position, adjusting the scanning parameter TE 100-120 ms. Due to the presence of fetal activity, during the scanning process, images collected at each layer were used as location reference for the scanning of the next layer. After the scan was completed, the image features were evaluated, and classification diagnosis for ACC cases was made.

Ultrasound diagnostic criteria [5]: Direct signs: Complete or partial absence of corpus callosum in the sagittal and coronal planes. Indirect signs: (1) The anterior horn and body of the lateral ventricle expanded outwards, and the bilateral ventricles were parallel; (2) The posterior horn of the lateral ventricle expanded ( $\geq 10 \mathrm{~mm}$ ), the anterior horn formed a horn peak, and the lateral ventricle was "Teardrop-like"; (3) The $3^{\text {rd }}$ ventricle showed different degrees of expansion and upward displacement; (4) Transparent septum disappeared; (5) Gyrus echo was presented between the lateral cerebral ventricle and falx cerebri. (6) Pericallosal artery subsidence was observed in ACC fetus from the sagittal section, with the loss of normal arc shape. Ultrasound suspected diagnosis for two of the above indirect signs was partial ACC.

MRI diagnostic criteria [6]: Direct signs: The corpus callosum of median sagittal plane was complete absence (complete ACC) (Fig. 1), partial absence and thinning of the corpus callosum (partial ACC) (Fig. 2); radial distribution of gyri, loss or absence of reed ball-shaped contour in the splenium.
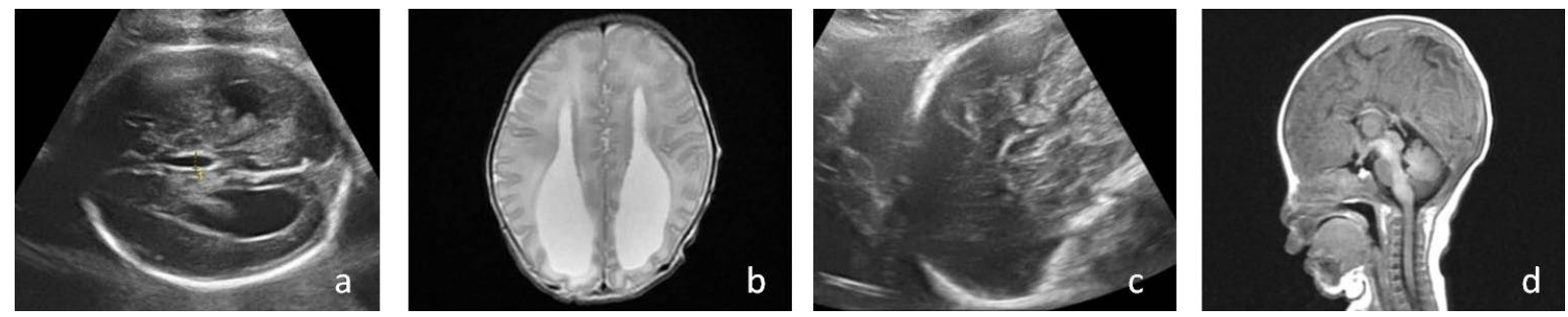

Figure 1. Complete ACC Ultrasound and MRI Comparison (for the same case); A. Ultrasonogram Cross-section vie; Lateral cerebral ventricle body outward expansion, loss of normal structure drawn towards the midline, the bilateral ventricles were parallel "Teardrop-like"; the posterior horn of the lateral ventricle expanded ( $\geq 10 \mathrm{~mm}$ ), the transparent septum disappeared, the $3^{\text {rd }}$ ventricle showed expansion and upward displacement, and the lateral ventricle was "Teardrop-like"; B. MRI image Axial view; The lateral ventricle anterior horn became smaller, with inverted " $\mathrm{A}$ " or crescentshaped separation, the lateral ventricle had near parallel separation, and the $3^{\text {rd }}$ ventricle showed expansion and upward displacement;

C. Ultrasonogram Longitudinal section view; The cerebral sulci appeared less clear, and gyri echo was not clearly seen; D. MRI image Sagittal vie; The corpus callosum was completely absent, and the gyrus showed radial distribution
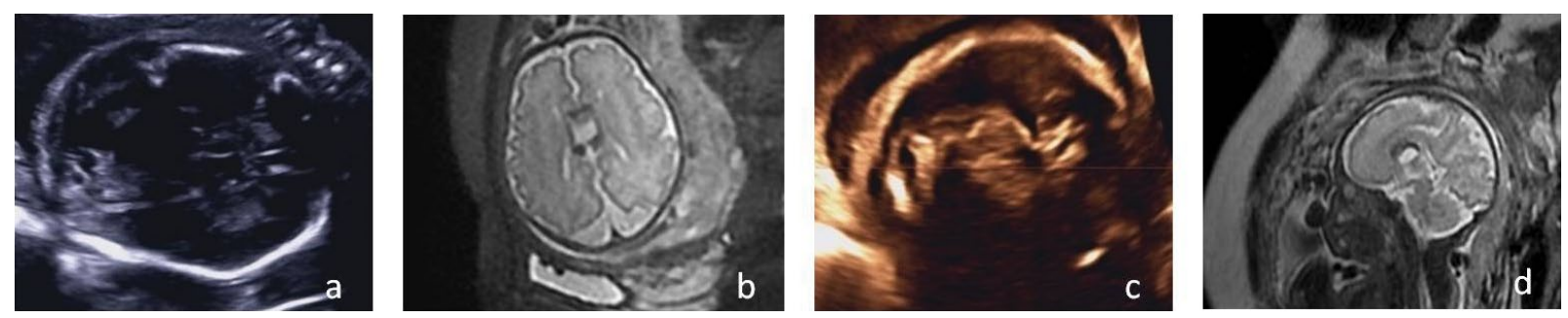

Figure 2. Partial ACC with Arachnoid Cyst Ultrasound and MRI Comparison (for the same case); A. Ultrasonogram Cross-section view; The transparent septum disappeared, the $3^{\text {rd }}$ ventricle showed expansion and upward displacement, cyst at left brain midline; B. MRI image Axial view; The transparent septum disappeared, cyst near posterior cranial midline, internal separation seen; $\mathbf{C}$. Ultrasonogram 3D sagittal section view; The rostrum and genu corporis callosi shown, caudomedial and splenium thinning, and cyst was not shown; D. MRI image Sagittal view; Cyst at midline of cranium, rostrum and genu corporis callosi shown, caudomedial and splenium thinning 

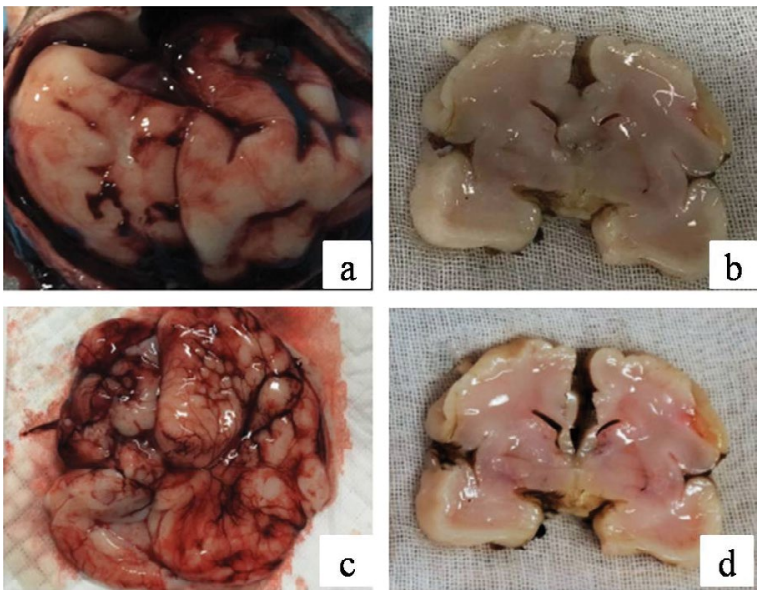

Figure 3. Autopsy chart; A. Partial ACC specimens have normal appearance and the brain channel is clear; $\mathbf{B}$. The anatomy of the pathological anatomy was edema, and the rear was thinning; $\mathbf{C}$. The overall appearance of the complete ACC specimen is uncoupled and unconnected; D. The corpus callosum is absent in pathology

Indirect signs: The lateral ventricle anterior horn became smaller, with inverted "A" or crescent-shaped separation; near parallel separation of the lateral ventricle; expansion of the trigone of lateral ventricles and posterior horn; the $3^{\text {rd }}$ ventricle showed expansion and upward displacement; fissurae interhemisphaerica was abnormally near the anterior part of the $3^{\text {rd }}$ ventricle directly or indirectly.

Chi-square test was used to analysis the consistency and difference of the results by SPSS 22.0.

\section{RESULTS}

In the 42 cases, from ultrasonic diagnosis, 18 cases were complete ACC and 24 cases were partial. From MRI diagnosis, 11 cases were complete $A C C, 16$ cases were partial. From the results after labor induction or birth, 11 cases were complete ACC, 14 cases were partial ACC, and rest were normal in the corpus callosum (Tab. 1).

The results should be verified again after birth or after labor induction.In the complete ACC, there were 7 cases of inconformity, 2 cases were partial ACC, 5 cases of callosum were normal. In partial ACC, only 14 cases were diagnosed and 10 cases were misdiagnosed.Ultrasound results show that the specificity, and diagnostic performance are poor and only applicable to the general screening of ACC (Tab. 2).

The results of partial ACC and complete ACC were identified with the results of after birth or labor induction. MRI results showed high sensitivity, strong specificity, strong diagnostic efficacy, high diagnostic value, and suitable for clinical diagnosis of ACC (Tab. 3).

In the 42 cases, 5 cases of brain malformations were misdiagnosis with prenatal ultrasonic diagnosis compared with MRI diagnosis in terms of concomitant intracranial malformations; and 2 cases of limb deformity and 4 cases of heart malformation were detected additionally by ultrasound compared with MRI diagnosis in terms of concomitant extracranial malformations (Fig. 1-3).

\section{DISCUSSION}

In this study, the sensitivity of ultrasound and MRI diagnosis of ACC were $100 \%$. While the specificity of ultrasound was $0 \%$, and MRI was $88.24 \%$; the positive predictive values were $59.52 \%$ and $92.59 \%$, respectively; the negative predictive values were 0 and $100 \%$; and the Youden indexes were 0 and 0.88 , respectively (Tab. $1-3$ ). The results showed that MRI had significant diagnostic value compared with prenatal ultrasound in the fetal ACC diagnosis. The reasons are analyzed as follows, firstly, the total number of cases is relatively small, and no cases of ultrasonic's result is normal but MRI diagnosis as ACC are found yet. In actual work, the

Table 1. Comparison of ultrasound and MRI diagnosis of different types of acc and results after birth or labor induction (unit: case)

\begin{tabular}{|l|l|l|l|}
\hline $\begin{array}{l}\text { Diagnostic } \\
\text { Classification }\end{array}$ & Ultrasound & MRI & $\begin{array}{l}\text { After Birth or } \\
\text { Laborlnduction }\end{array}$ \\
\hline Complete ACC & 18 & 11 & 11 \\
\hline Partial ACC & 24 & 16 & 14 \\
\hline Non ACC & 0 & 15 & 17 \\
\hline Total & 42 & 42 & 42 \\
\hline
\end{tabular}

$X^{2}=40.80 ; p<0.05$

Table 2. Sensitivity, specificity and diagnostic accuracy of ultrasonic examination

\begin{tabular}{|l|l|l|l|}
\hline \multirow{2}{*}{$\begin{array}{l}\text { Ultrasonic } \\
\text { Diagnosis }\end{array}$} & \multicolumn{2}{|l|}{$\begin{array}{l}\text { After Birth or Labor Induction (Gold } \\
\text { Standard) }\end{array}$} & \multirow{2}{*}{ Total } \\
\cline { 2 - 3 } & ACC & Normal & \\
\hline ACC & 25 & 17 & 42 \\
\hline Normal & 0 & 0 & 0 \\
\hline Total & 25 & 17 & 42 \\
\hline
\end{tabular}

Sensitivity to $\mathrm{ACC}, \mathrm{Se}=100 \%$, specificity $\mathrm{Sp}=0 \%$; Positive predictive value $+\mathrm{PV}=64.29 \%$; negative predictive value $-\mathrm{PV}=0$; Youden index $\mathrm{\gamma}=0$; diagnostic index $\mathrm{DI}=100 \%$

Table 3. Sensitivity, specificity and diagnostic accuracy of MRI examination

\begin{tabular}{|l|l|l|l|}
\multirow{2}{*}{$\begin{array}{l}\text { MRI } \\
\text { Diagnosis }\end{array}$} & \multicolumn{2}{|l|}{$\begin{array}{l}\text { After Birth or Labor Induction (Gold } \\
\text { Standard) }\end{array}$} & \multirow{2}{*}{ Total } \\
\cline { 2 - 3 } & ACC & Normal & \\
\hline ACC & 25 & 2 & 27 \\
\hline Normal & 0 & 15 & 15 \\
\hline Total & 25 & 17 & 42 \\
\hline
\end{tabular}

Sensitivity to ACC, Se $=100 \%$, specificity $\mathrm{Sp}=88.24 \%$; Positive predictive value $+\mathrm{PV}=92.59 \%$; negative predictive value $-\mathrm{PV}=100 \%$; Youden index $\gamma=0.88$; diagnostic index $\mathrm{DI}=188 \%$ 
cases that are completely normal in ultrasonic examination usually do not further go through MRI examination, hence there are defects in the inconsistent conditions for inclusion of the two method; next, in the diagnosis of ACC, especially partial ACC, ultrasound has the issue that the standard of diagnosis is too wide, and 11 cases of misdiagnosis are all suspected diagnosis only by the unclear transparent septum, third ventricle or lateral ventricular enlargement, which were confirmed as non-ACC by MRI and after birth examination. Another important reason is that it is very difficult to obtain the direct signs of the corpus callosum by ultrasound, and the image resolution is lower than MRI, the obtaining of indirect signs, for example, the lateral ventricle, the third ventricle, transparent septum and other changes may be caused by other brain malformations.

For the advantages of ultrasound relative to MRI in the blood flow imaging, due to the variable relative position of the fetal head and probe, the sensitivity of deep cerebral blood flow signal decreases, with reliability decreased significantly. The transvaginal ultrasound test is easier to show fetal coronal and sagittal section, but only in the fetal head position and when it is low. For the sagittal view shown by 3D imaging, this study shows that the image resolution still has a certain gap from the 2D ultrasound, the brainstem display rate is low, with the skull acoustic shadow, volume effect and other factors, and can only be used as reference. In addition to the above factors, the ultrasound image itself is influenced by the amniotic fluid, maternal body size, fetal skull, pelvic bone of the pregnant women and the ultrasound equipment limitations, and the result credibility is further reduced. In this study, it is considered that in the ultrasonic diagnosis of ACC, MRI shall be used as a necessary means of detection; if ultrasound cannot obtain satisfactory corpus callosum direct signs, the indirect signs need to meet at least three diagnostic criteria, with follow-up review, and the persistent presence of pathological signs, further MRI examination is performed, so as to reduce the misdiagnosis rate [7].

In this investigation, the diagnostic accuracy of complete ACC is significantly higher than that of partial ACC, indicating that ultrasound has relatively high diagnostic value for complete ACC. Among them, a total of 7 cases of complete ACC misdiagnosis (Tab. 1). Three cases were severe hydrocephalus compression of corpus callosum, transparent septum was not clearly shown, the deep cerebral blood flow signal was poor, resulting in ultrasound misdiagnosis as complete ACC. Two cases were encephalocele with partial ACC to intracranial structural changes, and ultrasound misdiagnosed for complete ACC with encephalocele. Two cases were partial ACC with concomitant septal dysplasia intracranial image changes misdiagnosed as complete ACC, which also shows that the diagnostic scale control of ultrasound in the complete ACC and partial ACC is inferior to MRI.
In this cohort, one case was diagnosed as partial ACC by both fetal prenatal ultrasound and MRI, and the postnatal MRI craniocerebral review was normal. Analysis of reasons show that, for fetal ACC when the gestational age is small ( 23 weeks of pregnancy in this case), the ultrasound and MRI resolution is lower than in a larger gestational age, whether partial ACC continues the development and improvement process in the uterus, so as to cause misdiagnosis. Therefore, when the gestational age is small, the diagnosis of ACC shall be prudent [8], while the corpus callosum development continues until adolescence. The early over diagnosis of partial ACC can easily increase the psychological burden of pregnant women, hence it should be cautious to make premature conclusions of partial ACC in the fetal period. In this study, it is considered that for complete ACC, ultrasound in the 24 weeks after pregnancy, MRI in the 26 weeks after pregnancy, the intracranial imaging changes are shown more clearly, and the ACC diagnosis has a higher consistency rate. For the diagnosis of partial ACC, ultrasound should be combined with MRI examination, and clear MRI diagnosis shall not be made until it is more than 32 weeks of pregnancy.

MRI detected four more cases of brain malformation than ultrasound, of which three cases were visual - septal dysplasia. In prenatal ultrasonography of severe septal dysplasia, the disappearance of clear septum was almost the only clue, which was easily confused with the ACC misdiagnosis. MRI could accurately identify the type and location of ACC, and could directly display the corpus callosum size, shape and degree of development and other intracranial structural abnormalities. On that account the central nervous system malformations, ultrasound has a greater misdiagnosis rate than MRI. In the extracranial malformation, this group of ultrasound and MRI can both identify the face, kidney, spine, digestive tract malformations, but has detected 2 more cases of limb deformity and 4 more cases of cardiac malformations than MRI. Analysis of the reasons shows that, the current rapid MRI imaging series only solve the problem of fetal movement, while rapid heart movement has not been treated with an appropriate technical means, and ultrasound blood flow imaging combined with structural abnormalities can be better detection of fetal heart malformations. In some limb abnormalities, as the ultrasound can be repeated observation of fetal limb posture changes to determine the more convenient conditions than MRI. Therefore, the current fetal MRI is more for intracranial and static soft tissue examination [9] relative to ultrasound, and ultrasound is currently superior in the above two examinations.

In the total 42 cases, 15 cases had chromosome examination, 5 cases were with chromosomal abnormalities, 3 cases were 18-trisomy, 1 case was 21-trisomy and 1 case 
was 13 -trisomy. The incidence of deformity was $28.57 \%$. In this group of ACC cases, 35 cases had induced labor, most were of other combined malformations (Fig. 3), isolated ACC due to the presence of the risk that the pregnant women could not undertake, the majority selected induction of labor. Seven cases of birth, and 1 case was diagnosed as partial ACC with interventricular septal defect with ultrasonic diagnosis. After birth and performed with MRI encephalic review, no significant intracranial abnormalities were seen, the prenatal diagnosis of ventricular septal defects were confirmed by the review, and in the follow-up currently. Five cases were false positive after birth without significant brain abnormalities by MRI review. One case was with visual-septal dysplasia, the neonatal visual acuity was low, and MRI review confirmed the prenatal MRI judgment.

\section{CONCLUSIONS}

In summary, in the diagnosis of fetal ACC, it is necessary to combine the ultrasonic examination with MRI examination, so as to avoid misdiagnosis. The consistency rate of complete ACC diagnosis is relatively high, while there is obvious limitation in the ultrasound diagnosis of partial ACC. There are obvious limitations in the diagnosis of partial ACC ultrasonography, so the diagnosis should be cautious. Although there are many deficiencies in prenatal ultrasound, due to its advantages of safe, economical, convenient, real-time imaging, repeatable check and the ability to detect the blood flow, it is still a good fetal development evaluation imaging method. The prenatal diagnosis of intracranial dysplasia with MRI is superior to that of prenatal ultrasound. The prenatal ultrasound has advantages in the fetal heart malformation, limb malformation diagnosis compared with
MRI. ACC is often accompanied by other malformations, so the ultrasonic diagnosis of ACC should be carefully observed to see if there are other malformations. Combined with MRI and chromosome examination, reliable diagnosis can be made, so as to provide an important basis for the decision making of good prenatal and postnatal care.

\section{REFERENCES}

1. Santo S, D'Antonio F, Homfray $T$, et al. Counseling in fetal medicine: agenesis of the corpus callosum. Ultrasound Obstet Gynecol. 2012; 40(5): 513-521, doi: 10.1002/uog.12315, indexed in Pubmed: 23024003.

2. Manevich-Mazor M, Weissmann-Brenner A, Bar Yosef O, et al. Added Value of Fetal MRI in the Evaluation of Fetal Anomalies of the Corpus Callosum: A Retrospective Analysis of 78 Cases. Ultraschall Med. 2018; 39(5): 513-525, doi: 10.1055/s-0043-113820, indexed in Pubmed: 29879745.

3. Santirocco M, Rodó C, Illescas T, et al. Accuracy of prenatal ultrasound in the diagnosis of corpus callosum anomalies. J Matern Fetal Neonatal Med. 2019 [Epub ahead of print]: 1-6, doi: 10.1080/14767058.2019.1609931, indexed in Pubmed: 31035852.

4. D'Antonio F, Pagani G, Familiari A, et al. Outcomes Associated With Isolated Agenesis of the Corpus Callosum: A Meta-analysis. Pediatrics. 2016; 138(3), doi: 10.1542/peds.2016-0445, indexed in Pubmed: 27581855.

5. Achiron R, Achiron A. Development of the human fetal corpus callosum: a high-resolution, cross-sectional sonographic study. Ultrasound Obstet Gynecol. 2001; 18(4): 343-347, doi: 10.1046/j.0960-7692.2001.00512.x, indexed in Pubmed: 11778993.

6. Rathee S, Joshi P, Kelkar A, et al. Fetal MRI: A pictorial essay. Indian J Radiol Imaging. 2016; 26(1): 52-62, doi: 10.4103/0971-3026.178326, indexed in Pubmed: 27081224.

7. Duczkowska A, Olwert A, Duczkowski M, et al. Postnatal verification of prenatal diagnoses established on foetal magnetic resonance imaging. Ginekol Pol. 2018; 89(5): 262-270, doi: 10.5603/GP.a2018.0045, indexed in Pubmed: 30084478.

8. Sotiriadis A, Makrydimas G. Neurodevelopment after prenatal diagnosis of isolated agenesis of the corpus callosum: an integrative review. Am J Obstet Gynecol. 2012; 206(4): 337.e1-337.e5, doi: 10.1016/j. ajog.2011.12.024, indexed in Pubmed: 22284958.

9. Peker N, Turan V, Ergenoglu M, et al. Assessment of total placenta previa by magnetic resonance imaging and ultrasonography to detect placenta accreta and its variants. Ginekol Pol. 2013; 84(3): 186-192, doi: 10.17772/gp/1561, indexed in Pubmed: 23700845. 